\title{
Common variable immunodeficiency in an 11-year-old girl - a case report
}

\author{
Joanna Pawłowska ${ }^{1, A-F}$, Olga Panowicz ${ }^{1, A-F} \oplus$, Joanna Jerzynska ${ }^{1, A-F} \oplus$ \\ ${ }^{1}$ Department of Paediatrics and Allergy, Medical University, Lodz, Copernicus Memorial Hospital, Korczak Paediatric \\ Centre, Lodz., Poland \\ A - Research concept and design, B - Collection and/or assembly of data, C - Data analysis and interpretation, \\ $D$ - Writing the article, E - Critical revision of the article, F - Final approval of article
}

Pawłowska J. Panowicz O. Jerzynska J. Common variable immunodeficiency in 11-year-old girl - a case report. J Pre Clin-Clin Res. 2020; 14(1): 14-17. doi: 10.26444/jpccr/119859

\begin{abstract}
Common variable immunodeficiency (CVID) is one of the most prevalent, heterogenous primary immunodeficiency syndromes in children and adults. Besides impaired immunoglobulin production by B cells, abnormalities in almost all components of the immune system have been desribed in CVID. The clinical pictures of CVID includes acute and chronic infections, inflammatory and autoimmune diseases, and can be associated with an increased incidence of cancer and lymphoma. Due to the plurality of clinical manifestations and different ages of occurance, diagnosis of CVID is difficult and belated in relation to primary symptoms. Patients with CVID require an integrated interprofessional team approach to minimalize complications of the disease and to improve patient outcomes. The article presents the clinical case of 11-yearold girl diagnosed with CVID in day-to-day medical practice.
\end{abstract}

\section{Key words}

immunodeficiency, autoimmunity, CVID, reccurent infections

\section{INTRODUCTION}

Common variable immunodeficiency (CVID) with a calculated prevalence of 1:10,000-1:50, 000 in Caucasians, and significantly less frequently in other populations, is one of the most rife symptomatic primary immunodeficiency in both adults and children $[1,2,3,4]$. The first symptoms may occur at different age, but two peaks of incidence have been noted: in childhood (between five and ten years old) and during the second and third decade of life. For his reason, CVID can be defined as either the belated onset of hypogammaglobulinaemia, or hypogammaglobulinaemia with two occurence peaks $[5,6]$. Patients affected by CVID are prone to reccurent infections, augmented in some reports by the occurence of autoimmune and lymphoproliferative diseases. The important issue at CVID is the variety of clinical symptoms which generates diagnostic difficulties, delays final diagnosis and thus the implementation of appropriate treatment which could protect patients from usually irreversible infectious and non-infectious complications [7]. Even though similarity concerning clinical and immunological characteristics can be observed in CVID patients, the severity and stage of progress differs remarkably among afflicted individuals [3]. Primary immunodeficiencies (PIDs) are connected with meaningful incidence and can lead to early decease if under diagnosed and untreated [8].

Address for cerrespondence: Joanna Pawłowska, Department of Paediatrics and Allergy, Medical University of Lodz, Copernicus Memorial Hospital, Korczak Paediatric Centre, Lodz. Poland

E-mail: asiap06@gmail.com

Received: 01.03.2020; accepted: 28.03.2020; first published: 31.03 .2020

\section{OBJECTIVE}

The aim of this paper is to emphasize the need to perform basic laboratory tests and to determine immunoglobulin classes in clinical practice in patients with recurrent infections. The abnormalities in these widely available tests should prompt extension of the diagnosis and allow for the early detection of disorders of the immune system.

\section{CASE REPORT}

An eleven-year-old female patient, delivered by c-section from the first pregnancy and born in the 39th week of pregnancy, with a body weight of $3,750 \mathrm{~g}$, Apgar score 10 , breastfed up to 15 months, remained under the care of the Department of Paediatrics and Allergy from the age of three. During early childhood, her development was evaluated as proper, weight and height rated at 70 centile on growth and body weight charts. Due to the Polish obligatory vaccination schedule, all vaccines were given and no supplemental inoculations administered. At the age of three, recurrent infections of both the upper and also lower respiratory system appeared, and the patient required very frequent administration of antibiotic therapy (at least once a month). Extensive alergological, laryngological and immunological diagnostics were implemented at that time. Results of performed laboratory tests revealed significantly decreased levels (compared to normal for age) of: IgG, IgM, IgG2 and IgG3 subclasses, CD19+ lymphocytes, inhibition of granulocyte activity in Bursttest, and also decreased levels of anti-Hbs titers. Elected serum specific IgE (aeroallergens and food allergens) were negative. Laryngological examination did not indicate any abnormalities.

On the basis of the clinical picture, laboratory tests and patient's age, the girl was diagnosed with hypogamma- 
globulinaemia and cellular immunity disorders. She was qualified to the national primary immunodeficiences treatment programme - IVIG replacement therapy. Monthly transfusions of immunoglobulins noticeably reduced the incidence of infections.

However, after a year, due to a renewed and increased prevalence of infections, the girl was admitted to hospitalization for further immunological evaluation. Examination of peripheral blood revealed significant deviations in immunological parameters: decreased levels of $\operatorname{IgA}$ and $\operatorname{IgM}$ in blood serum, reduced absolute count and percentage of lymphocyte $\mathrm{B}$, elevated percentage of lymphocytes T, mainly CD4+, normal percent and absolute count of NK cells at evaluation of individual subpopulations of lymphocytes. IgG persisted on a satisfactory level (the patient remained on immunoglobulins replacement therapy). After evaluation of the whole clinical history and elimination of other reasons for the hypogammaglobulinaemia, the patient was diagnosed with common variable immunodeficiency. During next four years, girl remained on IVIG replacement therapy; infections occurred, but relevantly less often.

In the next couple of months, the problem of chronic cough emerged. The suspicion of developing asthma occurred,and for that reason spirometry and skin prick tests were conducted, but both turned out to be negative. The cough infections did not always require antibiotic therapy, but pneumonia and obstructive bronchitis also occurred.

The patient underwent regular checkups, at least every six months, for immunological parameters, which revealed normal IgG and IgG subclasses level (immunoglobulin therapy was continued), but absolute count of lymphocytes B still remained relevantly decreased, with concurrent normal total count and percentage of NK cells and an elevated percentage of lymphocytes $\mathrm{T}$, particularly CD4+. IgA and IgM levels in blood serum were still significantly decreased, compared to age.

At the age of seven, the patient started a subcutaneous substitution of immunoglobulins, administered once a week in conditions of home therapy. Infections occurred occasionally. However, after two years, the frequency of respiratory tract infections increased - the girl needed repeated antibiotic therapy; she was admitted four times to the paediatric department due to pneumonia. Chronic antibiotic prophylaxis with amoxicillin (daily dose - 20 miligrams per kilogram per body weight) was instituted. The patient reported recurrent abdominal pain and periodically appearing diarrhoea; Giardia lamblia antigen was detected in faecal examination. Metronidazole was administered and after the treatment regression of symptoms was observed.

Due to the presence of reccurent dry cough, 24-hour $\mathrm{pH}$ monitoring with impedance was conducted. The patient was diagnosed with gastrooesophageal reflux; proton-pump inhibitor and continuous gastroenterological healthcare were advised.

Due to prominently increased incidence of infections and hospitalizations, despite the immunoglobulin replacement treatment, it has been decided to perform genetic tests which, at the time of writing this article, has been in the preparation.

The patients currently remains under interdisciplinary healthcare and the treatment with subcutaneous immunoglobulin substitution administered at home is continuing.

\section{DISCUSSION}

CVID is one of the most prevalent primary immunodeficiences (PIDs) with important morbidity and a high number of medical encounters [3,9]. Due to clinical and immune heterogeneity, CVID has been variably defined over the years [10]. In 2016, the International Consensus Document on CVID agreed on a definition requiring IgG levels two standard deviations below the age-appropriate reference, as well as either low IgA or IgM levels, and poor antibody response to vaccination in an individual at least 4 years-old, with no secondary cause of hypoglobulinaemia $[3,11]$.

The European Society of Immune Deficiences (ESID) diagnostic criteria are slightly different. To diagnose CVID, in addition to the laboratory abnormalities cited above, they require the presence of at least one of the following: increased susceptibility to infection, autoimmune manifestations, granulomatous disease, unexplained polyclonal lymphoproliferation, affected family member with antibody deficiency $[12,13]$. In all cases, other reasons for hypogammaglobulnaemia must be carefully excluded, including immune globulin loss, medications, viral infections, known immune defects or other medical reasons for hypogammaglobulinemia [10]. Because of physiologic immaturity, which can mimic CVID in the early years, the diagnose of CVID should not be established in children under the age of four $[13,14]$.

CVID affected patients are at increased risk of developing serious infections, not only bacterial, but also viral, parasitic and fungal. The most common infections, often appearing as the initial symptoms of an immune defect, are recurrent infections of the upper and lower respiratory tract, and sinusitis, which is often caused by capsular bacteria - Haemophilus influenzae t.b, Streptococcus pneumoniae, or atypical bacteria, such as Mycoplasma pneumoniae. Chronic and recurrent airway diseases, which can lead to bronchiectasis and pulmonary fibrosis, are the main cause of frequent hospitalizations, and incidence of mortality $[2,10,15,16]$. In CVID patients, gastrointestinal tract infections are also common, leading to recurrent or chronic diarrhea, and patients with CVID also have a higher prevalence of infections triggered by Giardia lamblia, Campylobacter, Salmonella sp., Helicobacter pylori, Cryptosporidium parvum, CMV and HBV, HCV [10, 14, 17].

Another common complication in CVID is autoimmunity (e.g. autoimmune thyroid disease or inflammatory bowel disease), affecting at least $25 \%$ of patients. Curiously, although not typical, this may be the first manifestation of an immune defect in an occasional patient who has never experienced significant clinical infection $[10,16,17,18,19]$.

Antibody-mediated cytopenia predominate in such cases, most often immune mediated thrombocytopenia (ITP), less frequently, haemolytic anaemia, or rarely, autoimmune neutropenia [10]. These patients are also significantly more likely to have one or more of other CVID-associated noninfectious complication, including lymphoproliferation, granulomatous disease, lymphoma, hepatic disease, interstitial lung diseases or enteropathy, as well as lymphadenopathy and splenomegaly $[5,10,17]$.

Patients with CVID also have an increased incidence of allergic diseases, with deteriorations and worse response to treatment, compared to individuals without PIDs (20).

A large number of CVID patients present a clinical history which may suggest allergic respiratory disease. There are 
studies which evaluate respiratory comorbidities in CVIDaffected individuals indicating the prevalence of asthma at the level of $15 \%$. Nevertheless, the role which is assigned to atopy in CVID-affected patients has not been determined unequivocally, because the total IgE serum level or specific IgE level are frequently not elevated. In these patients, it is possible that reports of asthma may be confused with a history of remittent respiratory system infections. There may also be difficulties in the evaluation of asthma in CVID patients, some of whom manifest respiratory symptoms as a cause of infectious complications, or may in fact be presentations of respiratory allergy [21].

Patients affected by CVID are susceptible to malignancy and have increased risk of developing cancers. The most frequent malignancy is non-Hodgin lymphoma, although other malignancies such as colorectal, breast and uterine cancer have been reported $[5,10,17,22]$.

The 11-year-old patient in this case report entirely fulfilled the diagnostic criteria for CVID, both laboratory - IgG, IgA and $\operatorname{IgM}<2 \mathrm{SD}$ of the normal levels for the age; poor response to vaccines, and clinical - increased susceptibility to infections and its recurrence and severity, gastroentero problems. There were no clinical signs, neither of autoimmunity nor lymphoproliferation and neoplastic disease, but the patient remains under multi-specialistic care to monitor potential symtoms and complications.

The treatment of humoral immune defect in CVID is based on immunoglobulin replacement therapy, at a dose $0.4-0.8 \mathrm{~g} / \mathrm{kg}$ intravenous once monthly $[5,23,24,25]$, or subcutaneous in an equivalent divided dose, with an interval of one to two weeks. Such treatment was also introduced in the presented case, initially intravenously then, after training the parents, by the subcutaneous route. Both routes of administration are efficacious, safe and widely available, being on the World Health Organization Essential Medicines Lists for adults and children [3]. Long-term administration of immunoglobulins protects against recurrence of severe and frequent infections [6], and was also effective in the presented case. Since enormous progress has been made in the genetic diagnosis of primary immunodeficiencies, the results are awaited of the genetic test in this patient.

CVID occurs very rarely in clinical practice, but it should not be missed as early diagnosis allows for rapid implementation of treatment, improvement in the clinical and quality-of-life outcomes, and avoidance of complications. Unfortunately, the average time from occurrence of the first symptoms until making an accurate diagnose is estimated at 4.4 years $[1,26]$. Reducing diagnostic delay, therefore, is crucial, and approaches include improved education and awareness, particularly regarding the onset and presentation of variable non-infectious of clinicians in both primary and secondary care. For that reason, it is especially important for paediatric general practitioners to have in mind, so called ' 10 warning signs of a primary immunodeficiency in children' (Tab. 1), which may suggest a primary immunodeficiency [27].

It is essential to determine if there is a family history of PID, draw attention to the current course and the rate of infection, effectiveness of their treatment, and evaluation of the physical and psychological development of the child.

Patients with CVID require an integrated inter-professional team approach to improve short-term and long-term patient outcomes. The main goal of therapeutic management in children with CVID is to decrease the morbidity and mortality
Table 1. If a paediatric patient is affected by at least 2 signs mentioned in the Table, there is a possible presence of an underlying primary immunodeficiency and the patient should be consulted with an immunologist

\begin{tabular}{ll}
\hline 4 ear infections in one year & $\geq 2$ deep-seated infections, including septicaemia \\
\hline $\begin{array}{l}\geq 2 \text { serious sinus infections } \\
\text { in one year }\end{array}$ & failure of an infant to gain weight or grow normally \\
\hline$\geq 2$ pneumonias in one year & family history of primary immuno-deficiency \\
\hline $\begin{array}{ll}\text { recurrent, deep skin or } & \begin{array}{l}\text { persistent thrush in mouth or fungal infection on } \\
\text { organ abscesses }\end{array}\end{array}$ \\
\hline
\end{tabular}

associated with recurrent infections. The consensus among paediatric immunologists is that close clinical monitoring and appropriate IgG replacement can ultimately extend the life expectancy of these young patients so that it approaches that of the general population [3].

\section{REFERENCES}

1. Grześk E, et al. Common variable immunodeficiency disorders diseases with many faces. Pediatr Pol 2018; 93(2): 186-191. doi: https:// doi.org/10.5114/polp.2..

2. Cooper CJ, Said S, Quansah R, et al. Pneumococcal meningitis in a young adult female with common variable immunodeficiency. Am J Case Rep 2013; 14: 471-475.

3. Bonilla FA et al. International Consensus Document (ICON): Common Variable Immunodeficiency Disorders. J Allergy Clin Immunol Pract 2016; 4: 38-59. doi: 10.1016/j.jaip.2015.07.025

4. Tseng CW, Lai KL, Chen DY, Lin CH, Chen HH. The incidence and prevalence of common variable immunodeficiency disease in Taiwan, a population-based study. PLoS ONE 2015; 10: e0140473.

5. Jolles $\mathrm{S}$. The variable in common variable immunodeficiency: a disease of complex phenotypes. J Allergy Clin Immunol Pract. 2013; 1: 545-556.

6. Abolhassani H, Sagvand BT, Shokuhfar T, et al. A review on guidelines for management and treatment of common variable immunodeficiency. Expert Rev Clin Immunol. 2013; 9: 561-574.

7. Szczawinska-Poplonyk A, Tapolska-Jozwiak K, Samara H. The B-cell compartment in antibody-deficient infants and young children developing common variable immunodeficiency or transient immune maturation? Ital J Pediatr. 2016; 42: 71.

8. Lankisch P, Schiffner J, Ghosh S, et al. The Duesseldorf warning signs for primary immunodeficiency: is it time to change the rules? J Clin Immunol. 2015; 35: 273-279.

9. Modell V, Quinn J, Orange J, Notarangelo LD, Modell F. Primary immunodeficiencies worldwide: an updated overview from the Jeffrey Modell Centers Global Network. Immunol Res. 2016; 64: 736-53.

10. Cunningham-Rundles Ch. Common Variable Immune Deficiency: Dissection of the Variable. Immunol Rev. 2019 January; 287(1): 145-161. doi: $10.1111 /$ imr.12728

11. Gereige JD, Maglione PJ. Current Understanding and Recent Developments in Common Variable Immunodeficiency Associated Autoimmunity. Front Immunol. 2019; 10: 2753. doi: 10.3389/ fimmu.2019.02753

12. Edgar D, Ehl S. ESID Registry - Working Definitions for Clinical Diagnosis of PID. 2016. p. 1-33.

13.http://esid.org/Working-Partie... (access: 17.01.2020).

14. Cunningham-Rundles $\mathrm{Ch}$. The many faces of common variable immunodeficiency. Hematology Am Soc Hematol Educ Program. 2012; 2012: 301-305. doi: 10.1182/asheducation-2012.1.301

15. Conaway E. Novel variant of common variable immunodeficiency. BMJ Case Rep 2017 May 27. doi: 10.1136/bcr-2017-219655

16. Patuzzo G, Barbieri A, Tinazzi E, et al. Autoimmunity and infection in common variable immunodeficiency (CVID). Autoimmun Rev. 2016; 15: 877-882. doi: 10.1016/j.autrev.2016.07.011

17. Cunningham-Rundles $C$. The many faces of common variable immunodeficiency. Hematology Am Soc Hematol Educ Program 2012; 2012: 301-305.

18. Genre J, Errante PR, Kokron CM, Toledo-Barros M, Câmara NOS, Rizzo LV. Reduced frequency of CD4+CD25HIGHFOXP3+ cells and diminished FOXP3 expression in patients with common variable immunodeficiency: a link to autoimmunity? Clin Immunol. 2009; 132: 215-21. doi: 10.1016/j.clim.2009.03.519 
19. Arandi N, Mirshafiey A, Abolhassani H, Jeddi-Tehrani M, Edalat R, Sadeghi B, et al. Frequency and expression of inhibitory markers of $\mathrm{CD} 4$ + CD25 + FOXP3 + regulatory T cells in patients with common variable immunodeficiency. Scand J Immunol. 2013; 77: 405-12. doi $10.1111 / \mathrm{sji} .12040$

20. Azizi G, Tavakol M, Rafiemanesh H, et al. Autoimmunity in a cohort of 471 patients with primary antibody deficiencies. Expert Rev Clin Immunol. 2017; 13: 1099-1106.

21. Agondi, et al. Allergic asthma in patients with common variable immunodeficiency. Allergy 2010; 65: 510-515.

22. Dhalla F, da Silva SP, Lucas M. Review of gastric cancer risk factors in patients with common variable immunodeficiency disorders, resulting in a proposal for a surveillance programme. Clin Exp Immunol. 2011; 165: 1-7.

23. Gathmann B, Mahlaoui N, Gérard L, et al. European Society for Immunodeficiencies Registry Working Party: Clinical picture and treatment of 2212 patients with common variable immunodeficiency. J Allergy Clin Immunol. 2014; 134: 116-126.

24. Gobert D, Bussel JB, Cunningham-Rundles C, et al. Efficacy and safety of rituximab in common variable immunodeficiency-associated immune cytopenias: a retrospective multicentre study on 33 patients. Br J Haematol. 2011; 155: 498-508.

25. Pac M, Bernatowska E. Comprehensive activities to increase recognition of primary immunodeficiency and access to immunoglobulin replacement therapy in Poland. Eur J Pediatr. 2016; 175: 1099-1105.

26. Mohammadinejad P, Aghamohammadi A, Abolhassani H, et al. Pediatric patients with common variable immunodeficiency: longterm follow-up. J Investig Allergol Clin Immunol. 2012; 22: 208-214.

27. Kalicki B, et al. Common variable immunodeficiency - case report. Centr Eur J Immunol. 2013; 38(1): 141-148. DOI: 10.5114/ceji.2013.34373 